\title{
Control interno y su incidencia en la gestión administrativa y financiera en la federación ecuatoriana de operadores y mecánicos de equipo caminero, (estudio de caso fedesomec, 2020)
}

\author{
MSc. Yadira Natalia Vergara Cuadros \\ Yadi.nat@hotmail.com \\ ORCID:0000-0003-0948-1595 \\ Empresa FEDESOMEC \\ MSc. Ramiro Enrique Guaman Chavez \\ ramiroguaman@tsachila.edu.ec \\ kenry.eg@hotmail.com \\ ORCID. 0000-0002-5593-4981 \\ Instituto Superior Tecnológico Tsa'chila \\ MSc. Paola Fernanda Mogollón Mena \\ pao_fer_mogo@hotmail.es \\ ORCID:0000-0001-5961-3539 \\ Instituto Superior Tecnológico Tsa'chila
}

\section{RESUMEN}

Esta investigación reveló la incidencia que tiene la aplicación del Control Interno en las diversas áreas de las entidades, sean estas privadas, públicas, anónimas, federaciones, compañías o sociedades; considerando, que su aplicación asegura que las actividades de una entidad se desarrollen eficazmente. Esta investigación tuvo el propósito de analizar minuciosamente el control efectuado por los miembros de la administración de FEDESOMEC e identificar puntos críticos que afectan el normal funcionamiento de la federación, además se determinó los factores de riesgo que inciden en la generación de información financiera y el cumplimiento de los indicadores de eficiencia, eficacia y economía, que influye en la toma de decisiones. La metodología que se aplicó es de carácter explicativa, descriptiva y exploratoria, las técnicas de investigación tales como la entrevista y encuesta; la entrevista se realizó al presidente nacional, contadora general y secretaria; la encuesta se la realizó a la totalidad de la población, y la observación directa se la ejecutará en todos los departamentos existentes en la entidad. Se espera que los resultados que se obtengan sobre el control interno incidente en la información financiera reflejen debilidades notables en los procedimientos. El sistema de control Interno Contable comprendido brinda muchos beneficios para FEDESOMEC un plan 
de organización, políticas, confiabilidad de los registros e información financieros, manuales de funciones, procedimientos; las operaciones se ejecuten de acuerdo con la autorización general o específica de la administración, donde se registren las operaciones como sean necesarias para permitir la mejor toma de decisiones en base a una información razonable.

Palabras claves: control interno contable; información financiera. 


\title{
Internal control and its impact on administrative and financial management in the ecuadorian federation of road equipment operators and mechanics, (case study fedesomec, 2020)
}

\begin{abstract}
This research revealed the incidence that the application of Internal Control has in the different areas of the entities, whether they are private, public, anonymous, federations, companies or societies; considering that its application ensures that the activities of an entity are developed efficiently. The purpose of this research was to thoroughly analyze the control carried out by the members of the administration of FEDESOMEC and to identify critical points that affect the normal operation of the federation, in addition to determining the risk factors that affect the generation of financial information and compliance with the indicators of efficiency, effectiveness and economy, which influences decision making. The methodology applied is of an explanatory, descriptive and exploratory nature, the research techniques such as interview and survey; the interview was conducted to the national president, general accountant and secretary; the survey was conducted to the entire population, and the direct observation will be carried out in all the existing departments of the entity. It is expected that the results obtained on the internal control over financial information will reflect notable weaknesses in the procedures. The internal accounting control system understood provides many benefits for FEDESOMEC an organizational plan, policies, reliability of financial records and information, function manuals, procedures; operations are executed in accordance with the general or specific authorization of the administration, where operations are recorded as necessary to allow the best decision making based on reasonable information.
\end{abstract}

Key words: internal accounting control; financial information.

Artículo recibido: 10 Agosto. 2021 Aceptado para publicación: 07. Setiembre. 2021

Correspondencia: Yadi.nat@ hotmail.com Conflictos de Interés: Ninguna que declarar 


\section{INTRODUCCIÓN}

La Federación Ecuatoriana de Operadores y Mecánicos de Equipos Camineros FEDESOMEC, se encuentra ubicada en la Provincia de Pichincha, Cantón Quito, Parroquia San Sebastián, Calle Ambato Oe2-283 y Guayaquil; fue creada con personalidad jurídica desde 1961, y está amparada en la Ley de Defensa Profesional y Ley de Desarrollo y Agricultura $\mathrm{N}^{\circ}$ 068, aprobada por la Honorable Asamblea Constituyente, el 9 de Junio de 1967; donde dispone a la federación de Capacitación de Operadores y Mecánicos de Equipo Caminero y otras obras similares FEDESOMEC, como la única entidad autorizada para realizar cursos de capacitación profesional, en todo el país Ecuador (LEXIS, 2012).

Dentro de FEDESOMEC se puede evidenciar el deficiente control en la gestión administrativa en el área financiera, que conlleva a realizar pagos innecesarios de glosas, multas y sanciones en la Federación Ecuatoriana de Operadores Mecánicos de Equipo Caminero FEDESOMEC. Además, se puede discernir el incremento del deficiente cumplimiento de normativas dificultando la ejecución de un proceso contable; de tal manera que se obstaculiza la aplicación de los procedimientos del área de forma eficiente y eficaz (Alaña, Gonzaga, y Yánez,2018).

En la gestión administrativa y financiera existe una débil estructura organizacional, este proceso da como resultado trabajos menos eficientes y uso incorrecto de recursos; repercute también tanto en la gestión administrativa, como desenvolvimiento del personal que labora en la FEDESOMEC; donde se desconocen sus responsabilidades, funciones y el orden jerárquico para reportar su trabajo y recibir órdenes (Cevallos, Moreno, y Chavez, 2020).

El deficiente control interno en la federación provocada por la inexistencia de métodos para garantizar la integridad de la información financiera y contable dentro de FEDESOMEC, esto genera que la información financiera no sea confiable, generalmente, debido a circunstancias imprevistas con riesgo de fraudes (Mendoza, Delgado, García y Barreiro, 2018).

El deficiente control a la gestión administrativa provoca incumplimiento de funciones y responsabilidades, ocasionando pérdida de tiempo y retraso para alcanzar los objetivos propuestos. Además, los reportes, impuestos y resultados ante las autoridades y entidades de control son generados de forma tardía e incompletos y esto genera multas, moras y si el caso lo amerita hasta clausura de la entidad (Moscoso, Tapia y Tapia, 2017)

Esta investigación está basada en; evaluar la incidencia que tiene el control interno en la gestión financiera y administrativa de la Federación Ecuatoriana de Operadores y Mecánicos 
de Equipo Caminero FEDESOMEC (Vivanco, 2017). La aplicación de este trabajo, del control interno y su incidencia en la gestión administrativa y financiera en FEDESOMEC, en el año 2020 mejorará la realización de cada tarea asignada a los empleados y la confiabilidad de las mismas, en especial del área contable; el sistema de control se encargará de regular las actividades a desarrollar en la empresa de acuerdo a las necesidades y exigencias a desempeñar, brindando un aporte significativo que sirva para la consecución de objetivos planteados, en bien de quienes pertenecen y dirigen la misma (Calle, Narváez y Erazo, 2020). El control interno facilita que FEDESOMEC pueda lograr sus metas y prestar sus servicios de manera eficaz, minimizando el factor riesgo y generar confianza a sus filiales ubicadas a nivel nacional; de tal manera que al aplicarlo se puede evaluar que los objetivos y metas trazadas sean cumplidas, que los directivos conozcan el desarrollo de la federación y los empleados mejoren su rendimiento y sean competitivos (Guamán, 2012).

\section{MÉTODOS Y MATERIALES}

El nivel o tipos de investigación que fueron utilizados fueron: descriptiva, explicativa, exploratoria, permitió el planeamiento del problema, a fin de tener una idea precisa de lo que se desea estudiar. Se la utilizó en la intervención a la federación para tener conocimiento del personal y con la situación que se encuentra la Federación, con el fin de conocer los problemas (Dáher, Panunzio, y Hernández, 2018).

Además, se utilizó métodos inductivo, deductivo, analítico y sintético, estos ayudaron a verificar la aplicación de normativas en cada operación que se realice en la FEDESOMEC, si se cumple o no con los indicadores de eficiencia, eficacia y economía, así mismo, con los métodos se lograron identificar la inexistencia de controles contables, y se pudo optimizar los recursos económicos. se consideró la totalidad de la población, que corresponde a personal administrativo y contable de la empresa donde se aplico una entrevista y cuestionario para obtener resultados optimos y precisos en esta investigación (Bernal,2016).

\section{ANÁLISIS Y DISCUSIÓN}

En la gestión administrativa - financiera de la Federación Ecuatoria de Operadores y Mecánicos de Equipo Caminero FEDESOMEC, presenta riesgos en el proceso de entrega de estados financieros, no se le realiza seguimiento en el cumplimiento de normas contables; Según, Warren, Warren, Reeve, \& Fees, (2016) define a la contabilidad financiera como un suministro de información a los individuos externos por medio de los balances económicos 
(p.4). es decir que es indispensable la presentación de balances donde indique la situación económica de la Federación, según políticas se la debe presentar semestralmente en reunión de comité nacional ordinaria para la debida toma de decisiones. El libro Contabilidad Financiera, sugiere que la contabilidad financiera es la encargada de registrar los movimientos financieros de una entidad, los reportes aportan información para los directivos y gestores (Sanín, 2015).

Al no contar con un manual de funciones y procedimientos en la gestión administrativa y financiera de la Federación, los empleados no tienen conocimientos de las actividades que deben desarrollar ni los procesos a seguir para su cumplimento. Un manual de procedimientos es el documento que contiene la descripción de actividades que deben seguirse en la realización de las funciones de una unidad administrativa, o de dos ò más de ellas (Silva y Silva, 2017). El manual incluye además los puestos o unidades administrativas que intervienen precisando su responsabilidad y participación. Los manuales de procedimientos establecen cómo se debe realizar determinada actividad con el objeto de obtener los más óptimos resultados, Tenorio, Tovar, \& Almeida, (2019).

Control interno para conocer su contribución en la gestión administrativa y financiera de la federación. 
Tabla 1.Cuestionario De Control Interno

\begin{tabular}{|c|c|c|c|}
\hline \multicolumn{4}{|c|}{ CUESTIONARIO DE CONTROL INTERNO } \\
\hline \multicolumn{4}{|c|}{ Componente: Ambiente de control } \\
\hline \multicolumn{4}{|c|}{$\begin{array}{l}\text { Nivel de Confianza } \\
\text { Calificación obtenida }\end{array}$} \\
\hline \multicolumn{4}{|c|}{$\begin{array}{c}\text { Nivel de confianza }=\frac{\text { Calificación obtenida }}{\text { Puntaje máximo }} \\
\text { Nivel de confianza }=\frac{53}{70}\end{array}$} \\
\hline \multicolumn{4}{|c|}{ Nivel de confianza $=0.76 \times 100=76 \%$} \\
\hline \multicolumn{4}{|c|}{$\begin{array}{c}\text { Nivel de Riesgo } \\
\text { Nivel de riesgo }=\text { Máximo puntaje }- \text { Nivel de confianza }\end{array}$} \\
\hline \multicolumn{4}{|c|}{ Nivel de riesgo $=100 \%-76 \%$} \\
\hline \multicolumn{4}{|c|}{ Nivel de riesgo $=24 \%$} \\
\hline NIVEL & BAJO & MODERADO & ALTO \\
\hline RIESGO & $0 \%-49 \%$ & $50 \%-75 \%$ & $76 \%-100 \%$ \\
\hline CONFIANZA & $0 \%-49 \%$ & $50 \%-75 \%$ & $76 \%-100 \%$ \\
\hline
\end{tabular}

Nota. Según la aplicación de cuestionario del COSO I en su componente Ambiente de Control, el presidente nacional de FEDESOMEC, indica que FEDESOMEC tiene un nivel de confianza alto correspondiente al $76 \%$ y un nivel de riesgo bajo del $24 \%$ por lo que no se brinda contrata personal profesional para brindar capacitación al personal, la capacitación se da entre trabajadores internos de FEDESOMEC. Fuente: Grupo Investigador, (2020) 
Tabla 2. Análisis de Nivel de Confianza y Riesgo

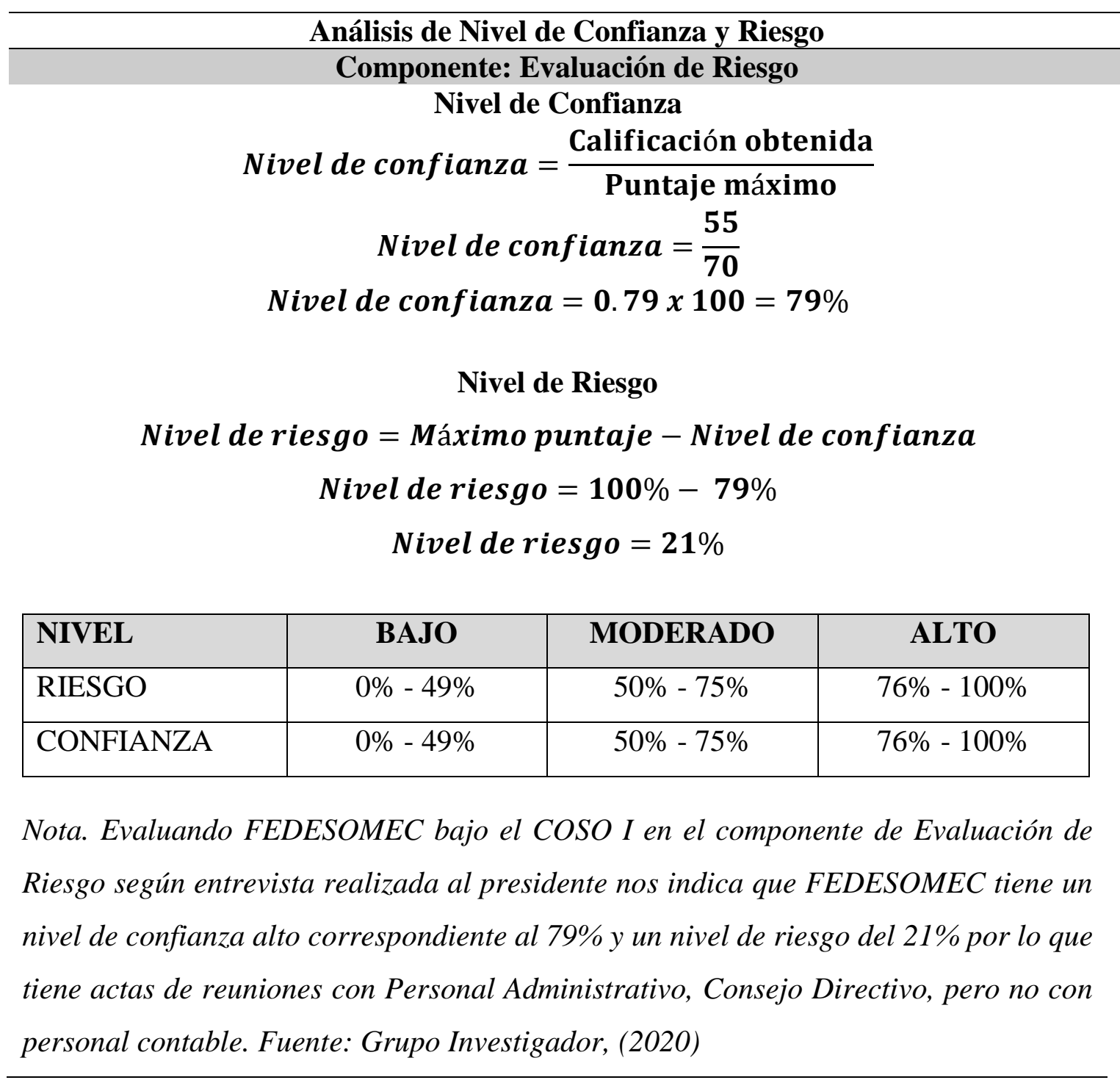


Tabla 3. Análisis de Nivel de Confianza y Riesgo

Análisis de Nivel de Confianza y Riesgo

\section{Componente: Actividades de control}

Nivel de Confianza

Nivel de confianza $=\frac{\text { Calificación obtenida }}{\text { Puntaje máximo }}$

Nivel de confianza $=\frac{\mathbf{5 0}}{\mathbf{5 0}}$

Nivel de confianza $=1 \times 100=100 \%$

Nivel de Riesgo

Nivel de riesgo $=$ Máximo puntaje - Nivel de confianza

$$
\begin{gathered}
\text { Nivel de riesgo }=100 \%-100 \\
\text { Nivel de riesgo }=0 \%
\end{gathered}
$$

\begin{tabular}{|l|c|c|c|}
\hline NIVEL & BAJO & MODERADO & ALTO \\
\hline RIESGO & $0 \%-49 \%$ & $50 \%-75 \%$ & $76 \%-100 \%$ \\
\hline CONFIANZA & $0 \%-49 \%$ & $50 \%-75 \%$ & $76 \%-100 \%$ \\
\hline
\end{tabular}

Nota. El COSO I fue creado con el fin de ayudar a mejorar los sistemas de control interno, es por esta razón que al ser empleado en FEDESOMEC mediante entrevista realizada al presidente en su componente Actividades de Control nos indica que FEDESOMEC tiene un nivel de confianza alto correspondiente al $100 \%$ y al no presentar riesgo en este componente es considerado bajo. Fuente: Grupo Investigador, (2020). 
Tabla 4. Análisis de Nivel de Confianza y Riesgo

\begin{tabular}{|c|c|c|c|}
\hline \multicolumn{4}{|c|}{ Análisis de Nivel de Confianza y Riesgo } \\
\hline \multicolumn{4}{|c|}{ Componente: Información y Comunicación } \\
\hline \multicolumn{4}{|c|}{ Nivel de Confianza } \\
\hline \multicolumn{3}{|c|}{ Nivel de confianza $=\frac{\text { Calificación obtenida }}{\text { Puntaje máximo }}$} & \\
\hline \multicolumn{4}{|c|}{ Nivel de confianza $=\frac{43}{50}$} \\
\hline \multicolumn{4}{|c|}{ Nivel de confianza $=0.86 \times 100=86 \%$} \\
\hline \multicolumn{4}{|c|}{ Nivel de Riesgo } \\
\hline \multicolumn{4}{|c|}{ Nivel de riesgo $=$ Máximo puntaje - Nivel de confianza } \\
\hline \multicolumn{4}{|c|}{ Nivel de riesgo $=100 \%-86 \%$} \\
\hline \multicolumn{4}{|c|}{ Nivel de riesgo $=14 \%$} \\
\hline NIVEL & BAJO & MODERADO & ALTO \\
\hline RIESGO & $0 \%-49 \%$ & $50 \%-75 \%$ & $76 \%-100 \%$ \\
\hline CONFIANZA & $0 \%-49 \%$ & $50 \%-75 \%$ & $76 \%-100 \%$ \\
\hline \multicolumn{4}{|c|}{$\begin{array}{l}\text { Nota. Utilizando el componente Información y Comunicación del COSO I, aplicada al } \\
\text { presidente de FEDESOMEC, refleja que la federación cumple con los requerimientos } \\
\text { indicados por tal razón tiene un nivel de confianza alto correspondiente al } 86 \% \text { y un } \\
\text { nivel de riesgo del } 14 \% \text { considerado bajo por que al momento de hacer seguimiento } \\
\text { se brinda solución, pero no documentado solo verbal. Fuente: Grupo Investigador, } \\
(2020) \text {. }\end{array}$} \\
\hline
\end{tabular}


Tabla 5. Análisis de Nivel de Confianza y Riesgo

\begin{tabular}{|c|c|c|c|}
\hline \multicolumn{4}{|c|}{ Análisis de Nivel de Confianza y Riesgo } \\
\hline \multicolumn{4}{|c|}{ Componente: Monitoreo o Supervisión } \\
\hline \multicolumn{4}{|c|}{ Nivel de Confianza } \\
\hline \multicolumn{4}{|c|}{ Nivel de confianza $=\frac{\text { Calificación obtenida }}{\text { Puntaje máximo }}$} \\
\hline \multicolumn{4}{|c|}{ Nivel de confianza $=\frac{40}{40}$} \\
\hline \multicolumn{4}{|c|}{ Nivel de confianza $=1 \times 100=100 \%$} \\
\hline \multicolumn{4}{|c|}{ Nivel de Riesgo } \\
\hline \multirow{2}{*}{\multicolumn{4}{|c|}{$\begin{array}{c}\text { Nivel de riesgo }=\text { Máximo puntaje }- \text { Nivel de confianza } \\
\text { Nivel de riesgo }=100 \%-100\end{array}$}} \\
\hline & & & \\
\hline \multicolumn{4}{|c|}{ Nivel de riesgo $=0 \%$} \\
\hline NIVEL & BAJO & MODERADO & ALTO \\
\hline RIESGO & $0 \%-49 \%$ & $50 \%-75 \%$ & $76 \%-100 \%$ \\
\hline CONFIANZA & $0 \%-49 \%$ & $50 \%-75 \%$ & $76 \%-100 \%$ \\
\hline
\end{tabular}

Nota. Aplicando cuestionario de COSO I en su componente de Monitoreo y Supervisión al presidente de FEDESOMEC nos da como resultados que es $100 \%$ confiable ya que no refleja riesgos en el monitoreo de actividades. Fuente: Grupo Investigador, (2020). 
Tabla 6. Consolidación de la evaluación del control interno del nivel de confianza y riesgo

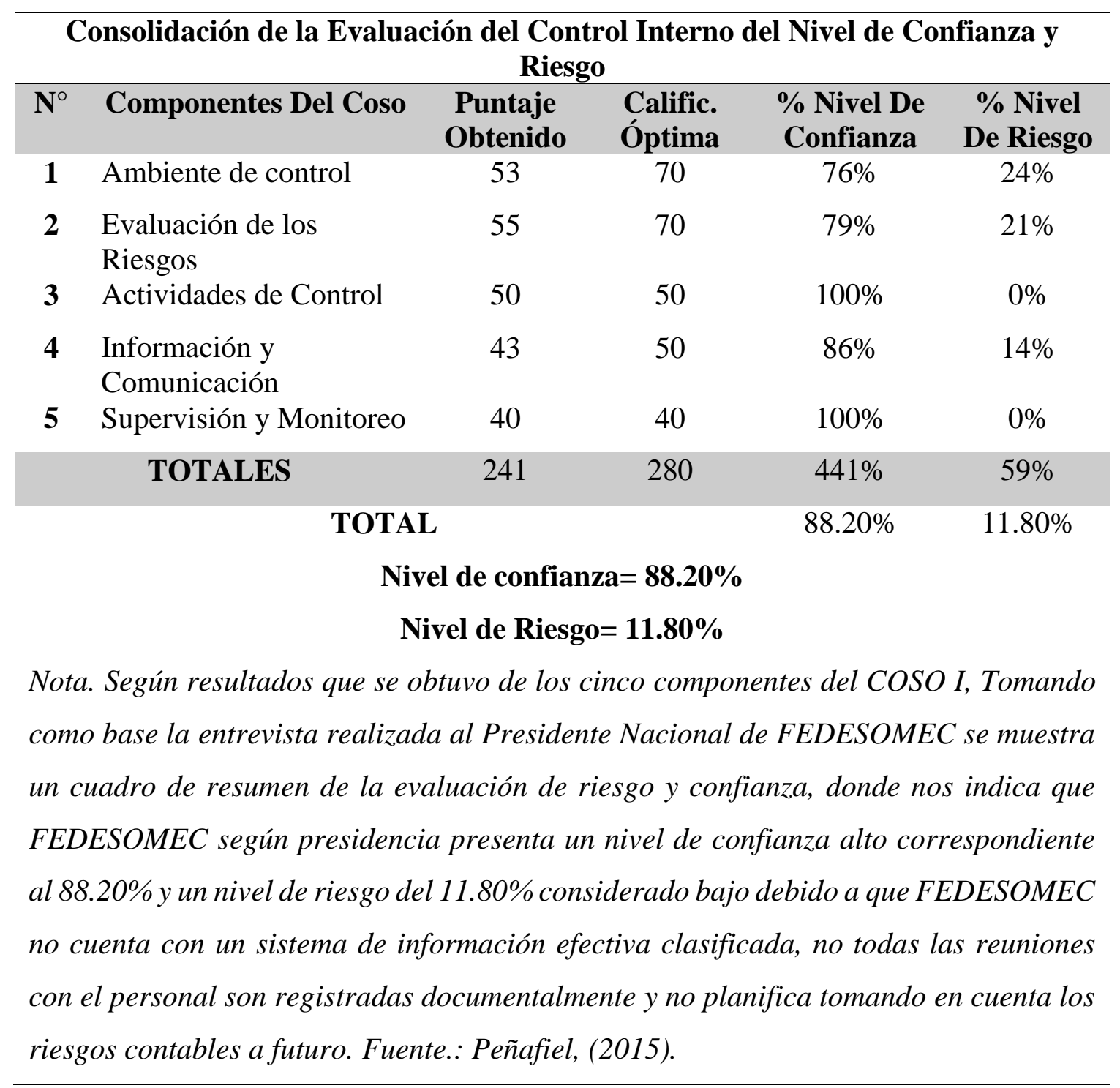


Tabla 7. Análisis de Nivel de Confianza y Riesgo

\begin{tabular}{l} 
Análisis de Nivel de Confianza y Riesgo \\
Componente: Ambiente de control \\
Nivel de Confianza \\
Nivel de confianza $=\frac{\text { Calificación obtenida }}{\text { Puntaje máximo }}$ \\
Nivel de confianza $=\frac{\mathbf{6 1}}{\mathbf{9 0}}$ \\
Nivel de confianza $=\mathbf{0 . 6 7} \boldsymbol{x} \mathbf{1 0 0}=\mathbf{6 7} \%$ \\
Nivel de Riesgo \\
Nivel de riesgo $=$ Máximo puntaje - Nivel de confianza \\
Nive riesgo $=\mathbf{1 0 0} \%$ - $\mathbf{6 7} \%$ \\
\hline NIVEL \\
\hline RIESGO
\end{tabular}

Nota. De acuerdo a la aplicación de la entrevista realizada a la Contadora General de FEDESOMEC en el componente ambiente de control según COSO I, refleja que FEDESOMEC tiene un nivel de confianza moderada correspondiente al $67 \%$ y nivel de riesgo bajo del $33 \%$ por lo que los reportes contables son presentados a los directivos cada seis meses de forma comparativa, además no existe un manual de funciones de empleados por escrito. Fuente: Grupo Investigador, (2020). 
Tabla 8. Análisis de Nivel de Confianza y Riesgo

\begin{tabular}{|c|c|c|c|}
\hline \multicolumn{4}{|c|}{ Análisis de Nivel de Confianza y Riesgo } \\
\hline \multicolumn{4}{|c|}{ Componente: Evaluación de Riesgo } \\
\hline \multicolumn{4}{|c|}{ Nivel de Confianza } \\
\hline \multicolumn{4}{|c|}{ Nivel de confianza $=\frac{\text { Calificación obtenida }}{\text { Puntaje máximo }}$} \\
\hline \multicolumn{4}{|c|}{ Nivel de confianza $=\frac{56}{60}$} \\
\hline \multicolumn{4}{|c|}{ Nivel de confianza $=0.93 \times 100=93 \%$} \\
\hline \multicolumn{4}{|c|}{ Nivel de Riesgo } \\
\hline \multicolumn{4}{|c|}{ Nivel de riesgo = Máximo puntaje - Nivel de confianza } \\
\hline \multicolumn{4}{|c|}{ Nivel de riesgo $=100 \%-93 \%$} \\
\hline \multicolumn{4}{|c|}{ Nivel de riesgo $=7 \%$} \\
\hline NIVEL & ВАJO & MODERADO & ALTO \\
\hline RIESGO & $\%-49 \%$ & $50 \%-75 \%$ & $76 \%-100 \%$ \\
\hline CONFIANZA & $\%-49 \%$ & $50 \%-75 \%$ & $76 \%-100 \%$ \\
\hline
\end{tabular}

Nota. Aplicando el COSO I en la federación mediante entrevista a la Contadora General de FEDESOMEC en el componente evaluación de riesgo nos indica que FEDESOMEC tiene un nivel de confianza alto correspondiente al $93 \%$ y nivel de riesgo bajo del 7\% por lo que, al existir retraso en los registros contables, se realiza una declaración de impuestos en cero para luego realizar la sustitutiva con valores correctos. Fuente: Grupo Investigador, (2020). 
Tabla 9. Análisis de Nivel de Confianza y Riesgo

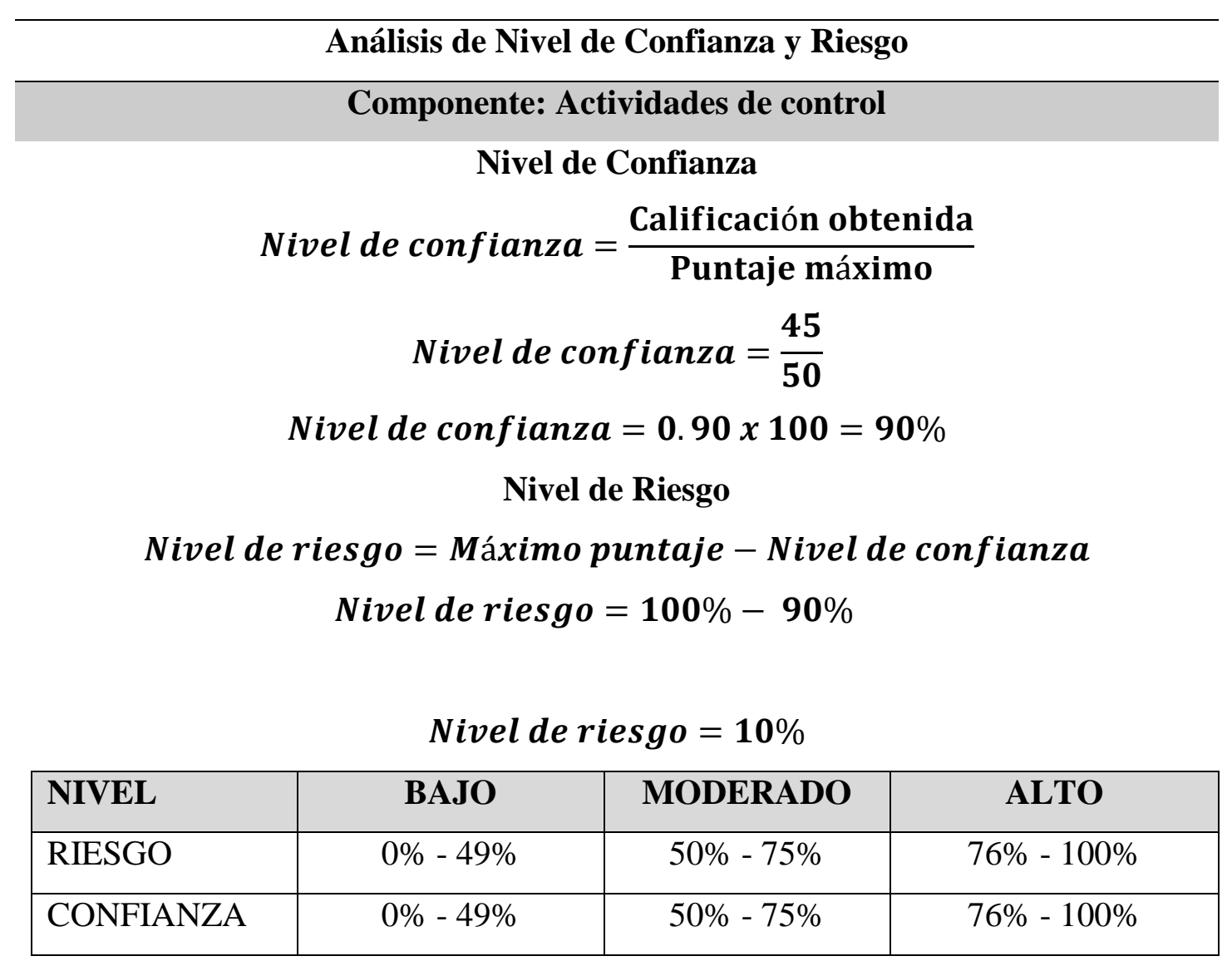

Nota. De la entrevista realizada a la Contadora General de FEDESOMEC en el componente actividades de control según COSO I, refleja que FEDESOMEC tiene un nivel de confianza alto correspondiente al $90 \%$ y nivel de riesgo bajo del $10 \%$ debido a que los registros contables no se realizan a diario si no tres días fijos a la semana. Fuente: Grupo Investigador, (2020). 
Tabla 10. Análisis de Nivel de Confianza y Riesgo

\begin{tabular}{|c|c|c|c|}
\hline \multicolumn{4}{|c|}{ Análisis de Nivel de Confianza y Riesgo } \\
\hline \multicolumn{4}{|c|}{ Componente: Información y Comunicación } \\
\hline \multicolumn{4}{|c|}{ Nivel de Confianza } \\
\hline \multicolumn{4}{|c|}{ Nivel de confianza $=\frac{\text { Calificación obtenida }}{\text { Puntaje máximo }}$} \\
\hline \multicolumn{4}{|c|}{ Nivel de confianza $=\frac{38}{40}$} \\
\hline \multicolumn{4}{|c|}{ Nivel de confianza $=0.95 \times 100=95 \%$} \\
\hline \multicolumn{4}{|c|}{ Nivel de Riesgo } \\
\hline \multirow{2}{*}{\multicolumn{4}{|c|}{$\begin{array}{c}\text { Nivel de riesgo }=\text { Máximo puntaje }- \text { Nivel de confianza } \\
\text { Nivel de riesgo }=100 \%-95 \%\end{array}$}} \\
\hline & & & \\
\hline \multicolumn{4}{|c|}{ Nivel de riesgo $=5 \%$} \\
\hline NIVEL & BAJO & MODERADO & ALTO \\
\hline RIESGO & $0 \%-49 \%$ & $50 \%-75 \%$ & $76 \%-100 \%$ \\
\hline CONFIANZA & $0 \%-49 \%$ & $50 \%-75 \%$ & $76 \%-100 \%$ \\
\hline
\end{tabular}

Nota. El COSO I tiene como objeto de ayudar a evaluar y mejorar sistemas de control interno, es por esta razón que mediante entrevista realizada a la Contadora General de FEDESOMEC se pretende conocer las debilidades y poder brindar sugerencias para mejorar, dando como resultado que FEDESOMEC tiene un nivel de confianza alto correspondiente al $95 \%$ y nivel de riesgo bajo del $5 \%$ debido a que no existe una persona fija para brindar solución a quejas y sugerencias, en cada departamento se encargan de dar solución. Fuente: Zapata, (2015). 
Tabla 11. Análisis de Nivel de Confianza y Riesgo

\begin{tabular}{|c|c|c|c|}
\hline \multicolumn{4}{|c|}{ Análisis de Nivel de Confianza y Riesgo } \\
\hline \multicolumn{4}{|c|}{ Componente: Monitoreo o Supervisión } \\
\hline \multicolumn{4}{|c|}{ Nivel de Confianza } \\
\hline \multicolumn{4}{|c|}{ Nivel de confianza $=\frac{\text { Calificación obt }}{\text { Puntaje máxì }}$} \\
\hline \multicolumn{4}{|c|}{ Nivel de confianza $=\frac{40}{40}$} \\
\hline \multicolumn{4}{|c|}{ Nivel de confianza $=1 \times 100=100 \%$} \\
\hline \multicolumn{4}{|c|}{ Nivel de Riesgo } \\
\hline \multicolumn{4}{|c|}{ Nivel de riesgo $=$ Máximo puntaje - Nivel de confianza } \\
\hline \multicolumn{4}{|c|}{ Nivel de riesgo $=100 \%-100 \%$} \\
\hline \multicolumn{4}{|c|}{ Nivel de riesgo $=0 \%$} \\
\hline NIVEL & BAJO & MODERADO & ALTO \\
\hline RIESGO & $0 \%-49 \%$ & $50 \%-75 \%$ & $76 \%-100 \%$ \\
\hline CONFIANZA & $0 \%-49 \%$ & $50 \%-75 \%$ & $76 \%-100 \%$ \\
\hline \multicolumn{4}{|c|}{$\begin{array}{l}\text { Nota. Concluida la entrevista realizada a la Contadora General de FEDESOMEC } \\
\text { según componente monitoreo y supervisión, nos indica que FEDESOMEC tiene un } \\
\text { nivel de confianza alto correspondiente al } 100 \% \text { y no existe nivel de riesgo. Fuente: } \\
\text { Grupo Investigador, (2020). }\end{array}$} \\
\hline
\end{tabular}


Tabla 12. Consolidación de la evaluación del control interno del nivel de confianza y riesgo.

\begin{tabular}{|c|c|c|c|c|c|}
\hline \multicolumn{6}{|c|}{$\begin{array}{l}\text { CONSOLIDACIÓN DE LA EVALUACIÓN DEL CONTROL INTERNO DEL } \\
\text { NIVEL DE CONFIANZA Y RIESGO }\end{array}$} \\
\hline $\mathbf{N}^{\circ}$ & $\begin{array}{c}\text { Componentes Del } \\
\text { Coso }\end{array}$ & $\begin{array}{l}\text { Puntaje } \\
\text { Obtenido }\end{array}$ & $\begin{array}{l}\text { Calific. } \\
\text { Óptima }\end{array}$ & $\begin{array}{l}\text { \% Nivel De } \\
\text { Confianza }\end{array}$ & $\begin{array}{c}\text { \% Nivel De } \\
\text { Riesgo }\end{array}$ \\
\hline 1 & Ambiente de control & 61 & 90 & 67 & 33 \\
\hline 2 & $\begin{array}{l}\text { Evaluación de los } \\
\text { Riesgos }\end{array}$ & 56 & 60 & 93 & 7 \\
\hline 3 & Actividades de Control & 45 & 50 & 90 & 10 \\
\hline 4 & $\begin{array}{l}\text { Información y } \\
\text { Comunicación }\end{array}$ & 38 & 40 & 95 & 5 \\
\hline 5 & $\begin{array}{l}\text { Supervisión y } \\
\text { Monitoreo }\end{array}$ & 40 & 40 & 100 & 0 \\
\hline & TOTALES & 240 & 280 & $445 \%$ & $55 \%$ \\
\hline & TOT & vivel de $R$ & $\begin{array}{l}z a=89.00 \\
g o=11 \%\end{array}$ & $89.00 \%$ & $11 \%$ \\
\hline $\begin{array}{l}\text { Not } \\
\text { com } \\
\text { un } \\
\text { FEL } \\
\text { al } 8 \\
\text { pres } \\
\text { mar }\end{array}$ & $\begin{array}{l}\text { Según resultados que se } \\
\text { base la entrevista realiz } \\
\text { ¿adro de resumen de la } \\
\text { ESOMEC según la conta } \\
\text { \% y un nivel de riesgo d } \\
\text { nta reportes mensuales } \\
\text { ra escrita si no en forma }\end{array}$ & $\begin{array}{l}\text { btuvo de lo } \\
\text { a a la Cont } \\
\text { aluación do } \\
\text { ra presentc } \\
11 \% \text { consic } \\
\text { los directi } \\
\text { erbal. Fuent }\end{array}$ & $\begin{array}{l}\text { inco com } \\
\text { ra Gener } \\
\text { esgo y co } \\
\text { nivel de } \\
\text { ado bajo } \\
\text { y el mar } \\
\text { Villada, L }\end{array}$ & $\begin{array}{l}\text { lentes del CO } \\
\text { le FEDESOM } \\
\text { anza, donde } \\
\text { afianza alto ce } \\
\text { ido a que FE } \\
l \text { de funcione } \\
\text { z y Muñoz, (2 }\end{array}$ & $\begin{array}{l}\text { O I, tomando } \\
\text { C se muestra } \\
\text { s indica que } \\
\text { respondiente } \\
\text { ESOMEC no } \\
\text { no existe de } \\
\text { 18). }\end{array}$ \\
\hline
\end{tabular}


Tabla 13. Análisis de Nivel de Confianza y Riesgo

\begin{tabular}{|c|c|c|c|}
\hline \multicolumn{4}{|c|}{ Análisis de Nivel de Confianza y Riesgo } \\
\hline \multicolumn{4}{|c|}{ Componente: Ambiente de control } \\
\hline \multicolumn{4}{|c|}{ Nivel de Confianza } \\
\hline \multirow{2}{*}{\multicolumn{4}{|c|}{ Nivel de confianza $=\frac{\text { Calificación obtenida }}{\text { Puntaje máximo }}$}} \\
\hline & & & \\
\hline \multicolumn{4}{|c|}{ Nivel de confianza $=\frac{44}{50}$} \\
\hline \multicolumn{4}{|c|}{ Nivel de confianza $=0.88 \times 100=88 \%$} \\
\hline \multicolumn{4}{|c|}{ Nivel de Riesgo } \\
\hline \multicolumn{4}{|c|}{ Nivel de riesgo = Máximo puntaje - Nivel de confianza } \\
\hline \multicolumn{4}{|c|}{ Nivel de riesgo $=100 \%-88 \%$} \\
\hline \multicolumn{4}{|c|}{ Nivel de riesgo $=12 \%$} \\
\hline NIVEL & BAJO & MODERADO & ALTO \\
\hline RIESGO & $\%-49 \%$ & $50 \%-75 \%$ & $76 \%-100$ \\
\hline CONFIANZA & $\%-49 \%$ & $50 \%-75 \%$ & $76 \%-100 \%$ \\
\hline
\end{tabular}

Nota. De acuerdo a los resultados según la secretaria de FEDESOMEC en el componente ambiente de control según COSO I, refleja que FEDESOMEC tiene un nivel de confianza alto correspondiente al $88 \%$ y nivel de riesgo bajo del $12 \%$ debido a que no existe en forma documental el código de ética y manual de funciones; se les indica a los empleados de forma verbal. Fuente: Grupo Investigador, (2020). 
Tabla 14. Análisis de Nivel de Confianza y Riesgo

\begin{tabular}{|c|c|c|c|}
\hline \multicolumn{4}{|c|}{ Análisis de Nivel de Confianza y Riesgo } \\
\hline \multicolumn{4}{|c|}{ Componente: Evaluación de Riesgo } \\
\hline \multicolumn{4}{|c|}{ Nivel de Confianza } \\
\hline \multicolumn{4}{|c|}{ Nivel de confianza $=\frac{\text { Calificación obtenida }}{\text { Puntaje máximo }}$} \\
\hline \multicolumn{4}{|c|}{ Nivel de confianza $=\frac{35}{40}$} \\
\hline \multicolumn{4}{|c|}{ Nivel de confianza $=0.875 \times 100=87.5 \%$} \\
\hline \multicolumn{4}{|c|}{ Nivel de Riesgo } \\
\hline \multicolumn{4}{|c|}{ Nivel de riesgo = Máximo puntaje - Nivel de confianza } \\
\hline \multicolumn{4}{|c|}{ Nivel de riesgo $=100 \%-87.5 \%$} \\
\hline \multicolumn{4}{|c|}{ Nivel de riesgo $=12.5 \%$} \\
\hline NIVEL & ВАJO & MODERADO & ALTO \\
\hline RIESGO & $0 \%-49 \%$ & $50 \%-75 \%$ & $76 \%-100 \%$ \\
\hline CONFIANZA & $0 \%-49 \%$ & $50 \%-75 \%$ & $76 \%-100 \%$ \\
\hline
\end{tabular}

Nota. De la presente entrevista realizada a la secretaria de FEDESOMEC en el componente evaluación de riesgo según COSO I, refleja que FEDESOMEC tiene un nivel de confianza alto correspondiente al $87.5 \%$ y nivel de riesgo bajo del $12.5 \%$ debido a que no existe en forma documental el código de ética y manual de funciones; se les indica a los empleados de forma verbal. Fuente: Grupo Investigador, (2020). 
Tabla 15. Análisis de Nivel de Confianza y Riesgo

\section{Análisis de Nivel de Confianza y Riesgo}

\begin{tabular}{|c|c|c|c|}
\hline \multicolumn{4}{|c|}{ Componente: Actividades de control } \\
\hline \multicolumn{4}{|c|}{ Nivel de Confianza } \\
\hline \multirow{2}{*}{\multicolumn{4}{|c|}{ Nivel de confianza $=\frac{\text { Calificación obtenida }}{\text { Puntaje máximo }}$}} \\
\hline & & & \\
\hline \multicolumn{4}{|c|}{ Nivel de confianza $=\frac{40}{50}$} \\
\hline \multicolumn{4}{|c|}{ Nivel de confianza $=0.80 \times 100=80 \%$} \\
\hline \multicolumn{4}{|c|}{ Nivel de Riesgo } \\
\hline \multirow{2}{*}{\multicolumn{4}{|c|}{$\begin{array}{c}\text { Nivel de riesgo }=\text { Máximo puntaje }- \text { Nivel de confianza } \\
\text { Nivel de riesgo }=100 \%-80 \%\end{array}$}} \\
\hline & & & \\
\hline \multicolumn{4}{|c|}{ Nivel de riesgo $=20 \%$} \\
\hline NIVEL & BAJO & MODERADO & ALTO \\
\hline RIESGO & $0 \%-49 \%$ & $50 \%-75 \%$ & $76 \%-100 \%$ \\
\hline CONFIANZA & $0 \%-49 \%$ & $50 \%-75 \%$ & $76 \%-100 \%$ \\
\hline
\end{tabular}

Nota. De los resultados de instrumentos aplicado a la secretaria de FEDESOMEC en el componente actividades de control según COSO I, refleja que FEDESOMEC tiene un nivel de confianza alto correspondiente al $80 \%$ y nivel de riesgo bajo del $20 \%$ debido a que a los empleados se les da a conocer de forma verbal sus funciones y entre compañeros se capacitan acerca de nuevos procesos. Fuente: Grupo Investigador, (2020). 
Tabla 16. Análisis de Nivel de Confianza y Riesgo

\begin{tabular}{|c|c|c|c|}
\hline \multicolumn{4}{|c|}{ Análisis de Nivel de Confianza y Riesgo } \\
\hline \multicolumn{4}{|c|}{ Componente: Información y Comunicación } \\
\hline \multicolumn{4}{|c|}{ Nivel de Confianza } \\
\hline \multicolumn{4}{|c|}{ Nivel de confianza $=\frac{\text { Calificación obtenida }}{\text { Puntaje máximo }}$} \\
\hline \multicolumn{4}{|c|}{ Nivel de confianza $=\frac{35}{40}$} \\
\hline \multicolumn{4}{|c|}{ Nivel de confianza $=0.875 \times 100=87.5 \%$} \\
\hline \multicolumn{4}{|c|}{ Nivel de Riesgo } \\
\hline \multicolumn{4}{|c|}{ Nivel de riesgo $=$ Máximo puntaje - Nivel de confianza } \\
\hline \multicolumn{4}{|c|}{ Nivel de riesgo $=100 \%-87.5 \%$} \\
\hline \multicolumn{4}{|c|}{ Nivel de riesgo $=12.5 \%$} \\
\hline NIVEL & BAJO & MODERADO & ALTO \\
\hline RIESGO & $0 \%-49 \%$ & $50 \%-75 \%$ & $76 \%-100 \%$ \\
\hline CONFIANZA & $0 \%-49 \%$ & $50 \%-75 \%$ & $76 \%-100 \%$ \\
\hline
\end{tabular}

Nota. Aplicando el COSO I a FEDESOMEC en su componente información y comunicación se realizó entrevista a la secretaria de la federación en el cual da como resultado que tiene un nivel de confianza alto correspondiente al $87.5 \%$ y nivel de riesgo bajo del $12.5 \%$ debido a que no existe capacitaciones con personas externas, entre compañeros se capacitan en procesos nuevos. Fuente: Grupo Investigador, (2020). 
Tabla 17. Análisis de Nivel de Confianza y Riesgo

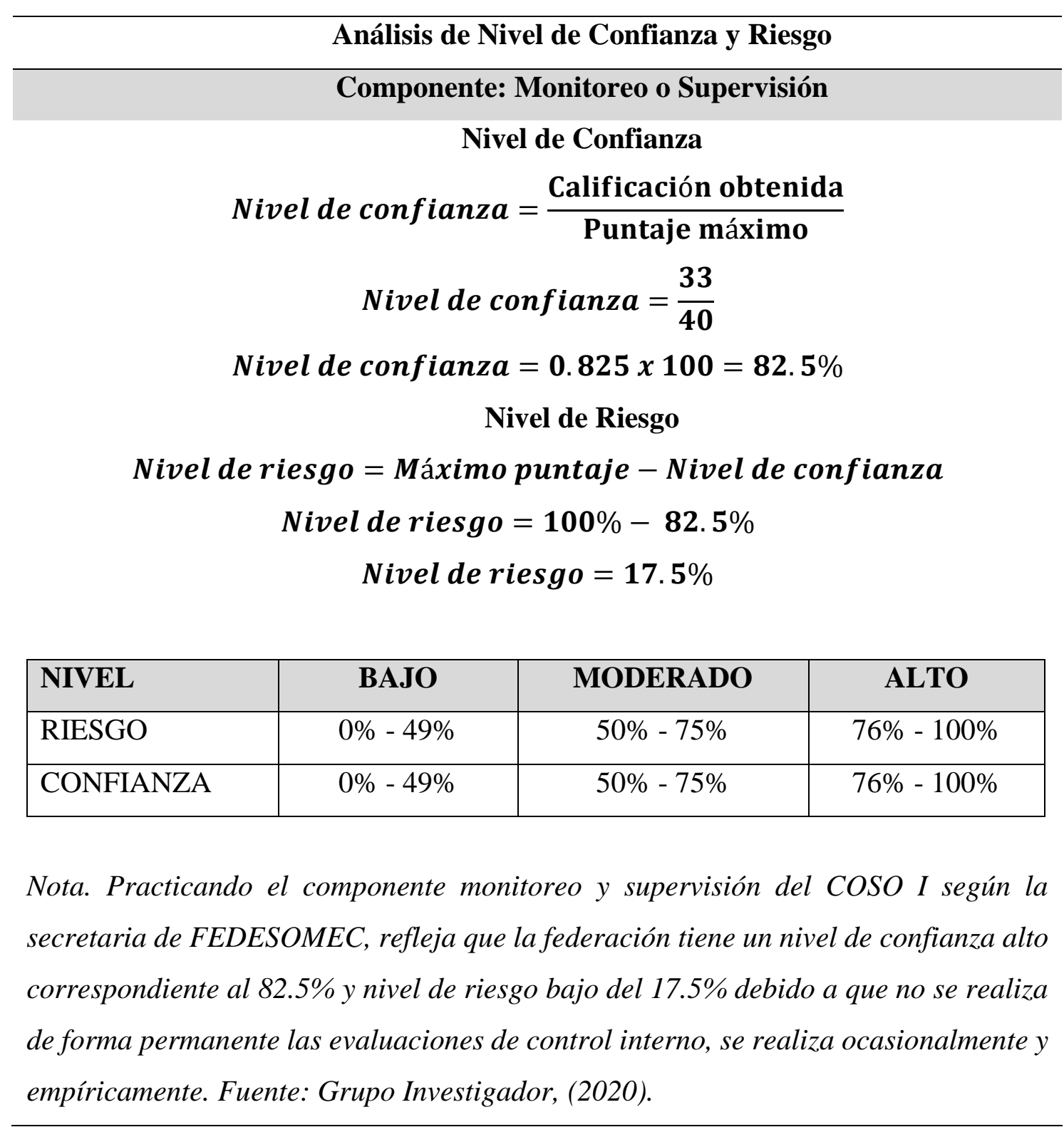


Tabla 18. Consolidación de la evaluación del control interno del nivel de confianza y riesgo

\begin{tabular}{c|l|r|r|c|c}
\hline \multicolumn{6}{c}{ Consolidación de la Evaluación del Control Interno del Nivel de } \\
Confianza y Riesgo \\
\hline $\mathbf{N}^{\circ}$ & $\begin{array}{l}\text { Componentes Del } \\
\text { Coso }\end{array}$ & $\begin{array}{c}\text { Puntaje } \\
\text { Obtenido }\end{array}$ & $\begin{array}{c}\text { Calific. } \\
\text { Óptima }\end{array}$ & $\begin{array}{c}\text { \% Nivel De } \\
\text { Confianza }\end{array}$ & $\begin{array}{c}\text { \% Nivel } \\
\text { De Riesgo }\end{array}$ \\
\hline 1 & $\begin{array}{l}\text { Ambiente de control } \\
2\end{array}$ & $\begin{array}{l}\text { Evaluación de los } \\
\text { Riesgos }\end{array}$ & 34 & $88 \%$ & $12 \%$ \\
\hline 3 & $\begin{array}{l}\text { Actividades de } \\
\text { Control }\end{array}$ & 40 & 40 & $87.5 \%$ & $12.5 \%$ \\
\hline 4 & $\begin{array}{l}\text { Información } \\
\text { Comunicación }\end{array}$ & 35 & 40 & $87.5 \%$ & $12.5 \%$ \\
\hline 5 & $\begin{array}{l}\text { Supervisión } \\
\text { Monitoreo }\end{array}$ & 33 & 40 & $82.5 \%$ & $17.5 \%$ \\
\hline \multicolumn{2}{c|}{ TOTALES } & $\mathbf{1 8 7}$ & $\mathbf{2 2 0}$ & $\mathbf{4 2 5 . 5 0}$ & $\mathbf{7 4 . 5 \%}$ \\
\hline
\end{tabular}

TOTAL

Nivel de confianza $=85.1 \%$

Nivel de Riesgo $=14.9 \%$

Nota. Planteando los cinco componentes del COSO I en FEDESOMEC; tomando como base la entrevista realizada a la secretaria se muestra un cuadro de resumen de la evaluación de riesgo y confianza, donde nos indica que FEDESOMEC según la secretaria presenta un nivel de confianza alto correspondiente al $85.10 \%$ y un nivel de riesgo del $14.90 \%$ considerado bajo debido a que FEDESOMEC no mantiene evaluaciones de control interno constante y no tiene manuales en forma documental si no verbal. Fuente: Grupo Investigador, (2020). 
Tabla 20. Cumplimiento de políticas

INDICADOR: Se lo realiza con el fin de identificar las políticas que se están cumpliendo.

$$
\begin{array}{r}
\text { CUMPLIMIENTO DE POLÍTICAS } \\
\% \text { Cumplimiento de políticas }=\frac{\text { Políticas Cumplidas }}{\text { Políticas Planteadas }} \\
\% \text { Cumplimiento de políticas }=\frac{3}{5} \\
\% \text { Cumplimiento de políticas }=\mathbf{0 . 6 0} \times 100=60 \%
\end{array}
$$

Nota. Los resultados obtenidos reflejan un nivel de efectividad de las políticas en un 60\% de cumplimiento. Fuente: Grupo Investigador, (2020).

INDICADOR: Para establecer el cumplimiento de las leyes que rigen a FEDESOMEC en el área administrativa y financiera.

\section{CUMPLIMIENTO DE LEYES}

$$
\begin{gathered}
\% \text { Cumplimiento de leyes }=\frac{\text { Leyes Cumplidas }}{\text { Leyes Planteadas }} \\
\% \text { Cumplimiento de leyes }=\frac{7}{7} \\
\% \text { Cumplimiento de leyes }=1 \times 100=100 \%
\end{gathered}
$$

Nota. Los resultados del cumplimiento de Leyes y Reglamentos se ubican en un 100\%, es decir que conforme a lo establecido FEDESOMEC ha cumplido de forma efectiva. Fuente: Grupo Investigador, (2020). 
Tabla 21. Cálculo de indicador de eficiencia $N^{\circ} 1$

DENOMINACIÓN:

Funciones y responsabilidades en el área de trabajo.

\section{FACTOR CRÍTICO:}

No contar con un manual de funciones y procedimientos documentados.

INDICADOR: Conocer las funciones que se están cumpliendo.

\section{CÁLCULO DE INDICADOR DE EFICIENCIA $\mathbf{N}^{\circ} 1$}

$\%$ Cumplimiento de funciones $=\frac{\text { funciones cumplidas }}{\text { funciones indicadas }}$

$$
\% \text { Cumplimiento de funciones }=\frac{23}{25}
$$

$$
\text { Cumplimiento de funciones }=0.92 \times 100=92 \%
$$

Nota. Los empleados cumplen en un 92\% las funciones indicadas en el desarrollo de sus labores en FEDESOMEC. Fuente: Grupo Investigador, (2020).

\section{Sugerencia:}

Establecer un manual de funciones, procedimientos por escrito e indicarles a los empleados según la función que desempeñan en FEDESOMEC. 
Tabla 22. Cálculo de indicador de eficiencia $N^{\circ} 2$.

DENOMINACIÓN:

$\begin{array}{ll}\text { Capacitaciones que recibe el personal } & \text { Los empleados no reciben } \\ \text { administrativo de FEDESOMEC. } & \begin{array}{l}\text { capacitaciones para su puesto de } \\ \text { trabajo de alguien externo. }\end{array}\end{array}$

INDICADOR: Establecer las capacitaciones que los empleados han recibido CÁLCULO DE INDICADOR DE EFICIENCIA $\mathrm{N}^{\circ} 2$

$$
\% \text { Capacitaciones a empleados }=\frac{20}{25}
$$

\section{Capacitaciones a empleados $=\mathbf{0 . 8 0} \times 100=80 \%$}

Nota. FEDESOMEC brinda en un $80 \%$ capacitaciones a los empleados, pero no contrata personas externas a FEDESOMEC para realizarlas, lo realizan entre compañeros de trabajo. Fuente: Grupo Investigador, (2020).

Sugerencia:

Contratar a capacitadores para actualizar conocimientos de los empleados y así puedan desenvolverse de una manera eficaz en sus labores.

Tabla 23. Cálculo de indicador de eficacia

\begin{tabular}{|c|c|}
\hline DENOMINACIÓN & FACTOR CRÍTICO \\
\hline $\begin{array}{l}\text { Respaldo digital y físico de la } \\
\text { información }\end{array}$ & $\begin{array}{l}\text { Falta de respaldo de documentos } \\
\text { en todas las áreas de FEDESOMEC }\end{array}$ \\
\hline $\begin{array}{l}\text { INDICADOR: Establecer la cantid } \\
\text { documentación existentes de FEDESOMEC. }\end{array}$ & \\
\hline
\end{tabular}

CÁLCULO DE INDICADOR DE EFICACIA

$\%$ Respaldo de documentación $=\frac{\text { documentos con repaldo }}{\text { total documentos }}$

$\%$ Respaldo de documentación $=\frac{10}{15}$

Respaldo de documentación $=0.67 \times 100=67 \%$ 
Nota. La federación realiza respaldos de la documentación física en un $67 \%$ es decir da cumplimiento con este indicador. Fuente: Grupo Investigador, (2020).

Sugerencia:

Adquirir hardware que permitan archivar la información y se logre mantener de forma óptima durante largo tiempo, a su vez realizar copias originales de la documentación física.

Tabla 24. cálculo de indicador de eficacia

DENOMINACIÓN:

Atención de quejas y sugerencias.

\section{FACTOR CRÍTICO:}

No existe una persona encargada de dar solución de quejas y sugerencias.

INDICADOR: Establecer la cantidad de quejas y sugerencias que se dan solución. CÁLCULO DE INDICADOR DE EFICACIA

$$
\begin{gathered}
\quad \% \text { Atención de quejas y sugerencias } \\
=\frac{\text { Quejas y sugerencias solucionadas }}{\text { total quejas y sugerencias }} \\
\quad \% \text { Atención de quejas y sugerencias }=\frac{14}{15}
\end{gathered}
$$

Atención de quejas y sugerencias $=0.93 \times 100=93 \%$

Nota. Al momento que los clientes presentan quejas y sugerencias, se le da un $93 \%$ de solución; a cada empleado que se le presenta la queja o sugerencia brinda solución. Fuente: Grupo Investigador, (2020).

\section{Sugerencia:}

Delegar a una persona que se encargue de dar seguimiento y solución a inconvenientes, por ende, a quejas y sugerencias que surgen de ellos.

Tabla 25. Cálculo de indicador de economía

\section{DENOMINACIÓN:}

Impuestos con instituciones entes de regulación.

\section{FACTOR CRÍTICO:}

Existe desorden en la fecha de pago de algunas obligaciones con entes reguladoras.

INDICADOR: Establecer la cantidad de obligaciones pagadas al día. 


\section{CÁLCULO DE INDICADOR DE ECONOMÍA \\ $\%$ Obligaciones pagadas a tiempo \\ $=\frac{\text { obligaciones canceladas a tiempo }}{\text { total obligaciones pagadas }}$ \\ $\%$ Obligaciones realizadas a tiempo $=\frac{6}{7}$}

Obligaciones realizadas a tiempo $=0.86 \times 100=86 \%$

Fuente. FEDESOMEC al ser una federación sin fines de lucro tiene obligaciones por pagar y en un $86 \%$ paga las obligaciones a tiempo. Fuente: Grupo Investigador, (2020).

Sugerencia:

Realizar un cronograma de fecha con valores de pagos aproximados de las obligaciones que se deben cancelar durante el mes y enviar a presidencia para tener el dinero con anticipación.

Tabla 26. Cálculo de indicador de economía

DENOMINACIÓN:

Impuestos declarados al SRI, ente de regulación.
FACTOR CRÍTICO:

Existencia de declaraciones con anomalías y valores pendientes de pago.

INDICADOR: Establecer la cantidad de impuestos que generan multas e interés en el año

\section{CÁLCULO DE INDICADOR DE ECONOMÍA}

$\%$ Impuestos que generan multas e interes $=\frac{\text { impuestos tardios }}{\text { total impuestos }}$

$\%$ Impuestos que generan multas e interes $=\frac{2}{15}$

Impuestos que generan multas e interes $=0.13 \times 100=13 \%$

Nota. Ante el ente regulador como es el SRI, IESS; se realizan impuestos y planillas mensuales, FEDESOMEC tiene un indicador de incumplimiento del 13\% ya que al no declararlas o darles solución a tiempo generan multas e intereses. Fuente: Grupo Investigador, (2020). 


\section{Sugerencia:}

Realizar y declarar los impuestos a tiempo mediante débito para evitar la generación de impuestos e intereses.

\section{RESUMEN DE INDICADORES}

\section{INDICADOR DE CUMPLIMIENTO}

$$
80 \%
$$

\begin{tabular}{|ccc|} 
& INDICADORES DE EFICIENCIA & \\
\hline $\mathbf{N}^{\circ} \mathbf{1}$ & $\mathbf{N}^{\circ} \mathbf{2}$ & PROMEDIO \\
\hline $\mathbf{9 2 \%}$ & $80 \%$ & $86 \%$ \\
\hline
\end{tabular}

\section{INDICADORES DE EFICACIA}

\begin{tabular}{|ccc|}
\hline $\mathbf{N}^{\circ} \mathbf{1}$ & $\mathbf{N}^{\circ} \mathbf{2}$ & PROMEDIO \\
\hline $\mathbf{6 7 \%}$ & $93 \%$ & $80 \%$ \\
& INDICADOR DE ECONOMÍA & \\
\hline $\mathbf{N}^{\circ} \mathbf{1}$ & $\mathbf{N}^{\circ} \mathbf{2}$ & PROMEDIO \\
$\mathbf{8 6 \%}$ & $87 \%$ & $86.5 \%$ \\
\hline
\end{tabular}

\section{RESUMEN DE INDICADORES}

$\begin{array}{ccccc}\text { CUMPLIMIENTO } & \text { EFICIENCIA } & \text { EFICACIA } & \text { ECONOMÍA } & \text { PROMEDIO } \\ \mathbf{8 0 \%} & 86 \% & 80 \% & 86.5 \% & 83.13 \%\end{array}$

Cumplimiento de indicadores $=\mathbf{8 3 . 1 3 \%}$

En el desarrollo de los indicadores de FEDESOMEC nos indica que tiene un cumplimiento del $83.13 \%$.

\section{CONCLUSIONES}

De la evaluación sobre la incidencia del control interno en la gestión administrativa y financiera de la Federación Ecuatoriana de Operadores y Mecánicos de Equipo Caminero FEDESOMEC, se determinó que existe una deficiencia en el control administrativo y financiero: 
Se puede mencionar que en la Federación no se cumplen a cabalidad las políticas internas y leyes que rigen la gestión administrativa y financiera, en especial las políticas correspondientes con el desarrollo intelectual y motivacional del personal que labora en oficinas de FEDESOMEC. Al aplicarse cuestionario de control interno mediante sus 5 componentes del COSO I se detectó que la Federación cumple con el $87.43 \%$ en sus responsabilidades y desarrollo de actividades administrativas y financieras, mientras que el $12.75 \%$ nos indica que existe falencias en el desenvolvimiento y desarrollo del personal; el presidente nacional tiene un nivel de incumplimiento de $11.80 \%$, la contadora con $11 \%$ y la secretaria con un $14.90 \%$; repercutiendo de forma externa a sus clientes y entes reguladores. Mediante la aplicación de indicadores de eficiencia, eficacia y economía en la gestión administrativa y financiera de la federación, da como resultado que se cumplen las políticas internas y leyes en un $80 \%$; el nivel de eficiencia se encuentra en un $86 \%$, eficacia en un $80 \%$ y economía en un 86.5\%; esto nos indica que FEDESOMEC, según indicadores tiene un nivel de cumplimiento en su gestión administrativa y financiera en un $83.13 \%$.

\section{REFERENCIAS BIBLIOGRAFICAS}

Alaña, T., Gonzaga, S., \& Yánez, M. (2018). Gestión Tributaria. Universidad y Sociedad, 10(2), 84-88. Obtenido de http://scielo.sld.cu/pdf/rus/v10n2/2218-3620-rus-10-0284.pdf

Dáher, J., Panunzio, A., \& Hernández , M. (2018). La investigación científica: una función universitaria a considerar en el contexto ecuatoriano. Revista EDUMECENTRO, 10(4), 1- 5. Obtenido de http://scielo.sld.cu/scielo.php?script=sci_arttext\&pid=S2077$28742018000400011 \& \operatorname{lng}=\mathrm{es} \& \mathrm{nrm}=$ iso.

Bernal, C. (2016). Metodología de la investigación (Cuarta ed.). Colombia, Colombia: PEARSON.

Calle , G., Narváez, C., \& Erazo, J. (2020). Sistema de control interno como herramienta de optimización de los procesos financieros de la empresa Austroseguridad Cía. Ltda. Revista Dominio de la ciencia, 6(1), 429-465. Obtenido de file://C:/Users/Ramiro\%20Guaman/Downloads/Dialnet-

SistemaDeControlInternoComoHerramientaDeOptimizaci-7351791.pdf

Cevallos , D., Moreno, C., \& Chavez, M. (2020). La Auditoría Interna. Revista Universidad y Sociedad, 10(5), 15-20. Obtenido de http://scielo.sld.cu/pdf/rus/v10n5/2218-3620rus-10-05-15.pdf 
Guamán , L. (11 de 04 de 2012). Diseño e implementación de un sistema de control interno en la Cooperativa de Ahorro y Crédito Innovación Andina. Tesis previo obtención titúlo de pregrado. Latacunga, Riobamba, Ecuador.

LEXIS. (25 de junio de 2012). Reglamento a Ley de Transporte Terrestre Transito y Seguridad Vial. Obtenido de www.obraspublicas.gob.ec: https://www.obraspublicas.gob.ec/wp-content/uploads/downloads/2015/03/DecretoEjecutivo-No.-1196-de-11-06-2012-REGLAMENTO-A-LA-LEY-DETRANSPORTE-TERRESTRE-TRANSITO-Y-SEGURIDAD-VIA.pdf

Mendoza, W., Delgado, M., García , T., \& Barreiro, I. (2018). El control interno y su influencia en la gestión administrativa del sector público. Revista Dominio de la Ciencia, 4(4), 206-240. Obtenido de file://C:/Users/Ramiro\%20Guaman/Downloads/Dialnet-

ElControlInternoYSuInfluenciaEnLaGestionAdministra-6656251.pdf

Peñafiel, K. (15 de Noviembre de 2015). Análisis de Gestión y Diseño de Procesos Aplicando el Informe Coso III para el Área de Talento Humano del "Instituto Tecnológico Superior Cordillera" en la Ciudad De D.M Quito. Tesis de pregrado de Universidad Central del Ecuador. Quito-Ecuador. Obtenido de http://www.dspace.uce.edu.ec/bitstream/25000/9373/1/T-UCE-0003-CA2922015.pdf

Rojas , M., Jaimes , L., \& Valencia , M. (2018). Efectividad, eficacia y eficiencia en equipos de trabajo. Revista Espacios, 39(6), 1-11. Obtenido de https://www.revistaespacios.com/a18v39n06/a18v39n06p11.pdf

Sanín, B. (2015). Administración de reyes (Primera ed.). Colombia : Made in Colombia Santillana, R. (2015). Sistema de Control Interno ( tercera edición ed.). Puebla , México : Pearson

Silva, V., \& Silva , L. (2017). Impacto de las salvaguardas a la importación de máquinas de coser periodo 2015-2016. Revista Dominio de la Ciencia, 3(2), 209-223. Obtenido de file://C:/Users/Ramiro\%20Guaman/Downloads/DialnetImpactoDeLasSalvaguardasALaImportacionDeMaquinasDe-6325857.pdf

Tenorio, J., Tovar, G., \& Almeida , O. (2019). Los manuales de procedimientos como base sólida de un emprendimiento. Revista FIPCAEC, 4(4), 194-210. Obtenido de 
file://C:/Users/Ramiro\%20Guaman/Downloads/109-

Texto\%20del\%20art\%C3\%ADculo-170-2-10-20191112.pdf

Villada , F., López , J., \& Muñoz , N. (2018). Análisis de la Relación entre Rentabilidad y Riesgo en la Planeación de las Finanzas Personales. Revista Formación universitaria, 11(6), 1-13. Obtenido de https://scielo.conicyt.cl/scielo.php?script=sci_arttext\&pid=S071850062018000600041

Vivanco Vergara, M. E. (2017). Los manuales de procedimientos como herramientas de control interno de una organización (Sexta ed.). Ecuador: Universidad y Sociedad.

Warren, Warren, C., Reeve, J., \& Fees, P. (2016). Contabilidad Financiera (Novena ed.). México: Coregraf Ixtapaluca.

Zapata , G. (2015). El tamaño y la estructura de la organización: un estudio teórico y empírico con el enfoque contingente. Revista Ciencias Estratégicas, 23(34), 193 - 208. Obtenido de https://www.redalyc.org/pdf/1513/151350864003.pdf 